MATHEMATICS OF COMPUTATION

Volume 70, Number 234, Pages 893-896

S 0025-5718(00)01178-9

Article electronically published on March 8, 2000

\title{
ON A CONJECTURE OF ERDŐS AND STEWART
}

\author{
FLORIAN LUCA
}

\begin{abstract}
For any $k \geq 1$, let $p_{k}$ be the $k$ th prime number. In this paper, we confirm a conjecture of Erdős and Stewart concerning all the solutions of the diophantine equation $n !+1=p_{k}^{a} p_{k+1}^{b}$, when $p_{k-1} \leq n<p_{k}$.
\end{abstract}

\section{INTRODUCTION}

For any $k \geq 1$ let $p_{k}$ be the $k$ th prime number. From [3], we found out that Erdös and Stewart conjectured that the only solutions of the equation

$$
n !+1=p_{k}^{a} p_{k+1}^{b} \quad \text { for some } a \geq 0, b \geq 0 \text { and } p_{k-1} \leq n<p_{k}
$$

are obtained for $n \leq 5$.

In this paper, we prove the following

Theorem. Equation (11) has no solutions for $n \geq 6$.

One can check that equation (1) has no solutions for $5<n \leq 11$. From now on, we work with a potential solution of (1) with $n \geq 12$.

\section{An ELEMENTARY LEMMA}

The following elementary result turns out to be helpful when searching for the values of $n$.

Lemma. In equation (1), one has $a b \neq 0$.

Proof of the Lemma. Assume that this is not so and write

$$
n !+1=p^{a} \quad \text { for some } p \in\left\{p_{k}, p_{k+1}\right\} .
$$

Let $a=2^{i} a_{1}$ where $a_{1} \geq 1$ is odd. Then,

(3) $\operatorname{ord}_{2}(n !)=\operatorname{ord}_{2}\left(p^{a}-1\right) \leq \max \left(\operatorname{ord}_{2}(p \pm 1)\right)+i \leq \log _{2}\left(p_{k+1}+1\right)+\log _{2}(a)$.

From equation (2), we know that

$$
n^{a}<p^{a}=n !+1<n^{n},
$$

therefore $a<n$. Since the interval $[n+1,2 n]$ contains at least two primes for $n \geq 12$, we get $p_{k+1}+1 \leq 2 n$. Hence, inequality (3) implies

$$
\operatorname{ord}_{2}(n !)<\log _{2}(2 n)+\log _{2}(n)=2 \log _{2}(n)+1 .
$$

Received by the editor January 4, 1999.

2000 Mathematics Subject Classification. Primary 11D61.

Key words and phrases. p-adic linear forms in two logarithms.

(C)2000 American Mathematical Society 
From Lemma 1 in [1, we know that

$$
\operatorname{ord}_{2}(n !) \geq n-\log _{2}(n+1) .
$$

From inequalities (5) and (6), we get

$$
n-\log _{2}(n+1)<2 \log _{2}(n)+1,
$$

which implies $n \leq 11$. This contradicts the assumption on $n \geq 12$.

\section{A LiNEAR FORM IN LOGARITHMS AND A BOUND ON $n$}

Write

$$
n !=p_{k}^{a} p_{k+1}^{b}-1=p_{k+1}^{b}\left(p_{k}^{a}-\left(\frac{1}{p_{k+1}}\right)^{b}\right) .
$$

We find an upper bound for $\operatorname{ord}_{2}(n !)$. We apply Théoréme 4 in 1 with the choices

$$
\begin{gathered}
p=2, \quad D=1, \quad g=1, \\
\alpha_{1}=p_{k}, \quad \alpha_{2}=\frac{1}{p_{k+1}}, \quad b_{1}=a, b_{2}=b, \\
A_{1}=p_{k}, A_{2}=p_{k+1}
\end{gathered}
$$

and

$$
\mu=15, \quad \nu=10, \quad c(\mu, \nu)=18 .
$$

From the result in 1, it follows that

(9) $\operatorname{ord}_{2}(n !) \leq \frac{36}{(\log 2)^{4}}\left(\max \left\{\log b^{\prime}+\log \log 2+0.4,15 \log 2\right\}\right)^{2} \log p_{k} \log p_{k+1}$,

where

$$
b^{\prime}=\frac{a}{\log p_{k+1}}+\frac{b}{\log p_{k}} .
$$

We now find a bound on $b^{\prime}$ in terms on $n$. Since

$$
p_{k}^{a} p_{k+1}^{b}=n !+1<n^{n},
$$

it follows that

$$
a \log p_{k}+b \log p_{k+1}=\log p_{k}^{a} p_{k+1}^{b}<\log n^{n}=n \log n .
$$

Hence,

$$
b^{\prime}=\frac{a}{\log p_{k+1}}+\frac{b}{\log p_{k}}=\frac{a \log p_{k}+b \log p_{k+1}}{\log p_{k} \log p_{k+1}}<\frac{n \log n}{\log p_{k} \log p_{k+1}}<\frac{n}{\log n} .
$$

Since the interval $[n+1,2 n]$ contains at least two primes, it follows that $p_{k}<$ $p_{k+1}<2 n$. Inequality (9) now implies

$$
\operatorname{ord}_{2}(n !)<\frac{36}{(\log 2)^{4}}\left(\max \left\{\log \left(\frac{n}{\log n}\right)+\log \log 2+0.4,15 \log 2\right\}\right)^{2} \log ^{2}(2 n) .
$$

When

$$
\log \left(\frac{n}{\log n}\right)+\log \log 2+0.4 \leq 15 \log 2,
$$


we get $n<409506$. When

$$
\log \left(\frac{n}{\log n}\right)+\log \log 2+0.4>15 \log 2,
$$

we get, by inequalities (6) and (13), that

$$
n-\log _{2}(n+1)<\frac{36}{(\log 2)^{4}}\left(\log \left(\frac{n}{\log n}\right)+\log \log 2+0.4\right)^{2} \log ^{2}(2 n),
$$

which implies $n<7242116$. The conclusion is that $n<p_{k}<p_{k+1}<7.5 \cdot 10^{6}$.

\section{The Remaining COMPutations}

For the remaining computations, we used the following result due to Erdős and Obláth (see [2]).

Theorem EO. The equation

$$
x^{p} \pm y^{p}=n !
$$

has no solutions such that $p>2$ is prime and $\operatorname{gcd}(x, y)=1$.

Case 1. $n>193$.

The idea here was to check, computationally, that if $n$ leads to a solution of (1), then $a \equiv b \equiv 0(\bmod 3)$. Once we prove this, the impossibility of (1) follows from Theorem EO for $p=3$.

Assume, for example, that (1) has a solution such that either $3 \nmid a$ or $3 \nmid b$. Write

$n !+1=A x^{3} \quad$ where $A=p_{k}^{\delta_{1}} p_{k+1}^{\delta_{2}}$ for some $\delta_{1}, \delta_{2} \in\{0,1,2\}$ with $\left(\delta_{1}, \delta_{2}\right) \neq(0,0)$.

Let $q \leq 193$ be a prime congruent to 1 modulo 3. Equation (11) implies that $A x^{3} \equiv 1(\bmod q)$ for every such $q$. It now follows that $A$ is a cubic residue modulo $q$ for every $q \leq 193$ which is congruent to 1 modulo 3 . Since a number $y$ is a cubic residue modulo $q$ if and only if $y^{2}$ is a cubic residue modulo $q$, it follows that we need to identify only those numbers $A$ of the form

$$
A=p_{k} \quad \text { or } \quad A=p_{k} p_{k+1} \quad \text { or } \quad A=p_{k}^{2} p_{k+1}
$$

in the range $193<p_{k}<p_{k+1}<7.5 \cdot 10^{6}$ which are cubic residues with respect to every prime $q \leq 193$ which is congruent to 1 modulo 3. Achim Flammenkamp wrote a computer program which checked in a few minutes that there are no such $A$ 's. Hence, $n \leq 193$.

Case 2. $n \leq 193$.

By the Lemma, we know that if $n$ leads to a solution of (1), then $a b>0$. Achim Flammenkamp wrote another computer program which checked in less than a second that in this range $n !+1 \not \equiv 0\left(\bmod p_{k} p_{k+1}\right)$.

The Theorem is therefore proved.

\section{ACKNOWLEDGMENTS}

We thank Achim Flammenkamp who carried out the computations described in the last section. We also thank Professor Andreas Dress and his research group in Bielefeld for their hospitality during the period when this paper was written, and the Alexander von Humboldt Foundation for support. 


\section{REFERENCES}

[1] Y. Bugeaud \& M. Laurent, Minoration effective de la distance p-adique entre puissances de nombres algébriques, J. Number Theory 61 (1996), 311-342. MR 98b:11086

[2] P. Erdős \& R. Obláth, Über diophantische Gleichungen der Form $n !=x^{p} \pm y^{p}$ und $n ! \pm m !=$ $x^{p}$, Acta Szeged 8 (1937), 241-255.

[3] R. K. Guy, Unsolved problems in number theory, Springer-Verlag, New York, 1994, Problem A2. MR 96e:11002

Mathematical Institute, Czech Academy of Sciences, Z̆itná 25, 11567 Praha 1, Czech REPUBLIC

E-mail address: luca@math.cas.cz 